\title{
Hinweise auf japanische Energiefragen
}

Als Mass für den wirtschaftlichen Entwicklungsstand eines Landes wird oft der Verbrauch an Primärenergie : Einwohner verwendet. Dieser Wert ist verhältnismässig einfach zu berechnen; ausserdem ist er hoch mit anderen Masszahlen (z.B.dem BSP:Kopf) korreliert. Die entsprechende Zuwachsrate betrug in Japan in den Fünfzigerjahren 7, in den Sechzigerjahren 10 Prozent. 1974 wurde mit $3839 \mathrm{~kg}$ SKE (Steinkohleeinheiten) ein Verbrauch erreicht, der jenem der europäischen Industrieländer gleichkam. Diese Tatsache wird in Japan mit Befriedigung registriert.

1950 konnte fast der gesamte Bedarf an Primärenergie im Lande selbst gewonnen werden; 1974 waren es nur noch $8,5 \%$. Die Auslandabhängigkeit stieg bei der Energieversorgung ständig und betrug nach Angaben des Ministry of International Trade and Industry (MITI) 1960 44, 2 und 1973 98, 9 Prozent. Bisher stellten sich kaum $\mathrm{Be}-$ schaffungsprobleme, auch wenn man in Japan 1973 eine Verknappung von Erdöl erwartete. Trotz Oelkriese betrug mengenmässig die Einfuhr von Erdöl 1974 96\% im Vergleich mit 1973. Empfindlich wirkte sich dagegen die Preissteigerung aus:Trotz praktisch gleicher Einfuhrmenge betrug der Einfuhrwert 1974 3,4 mal mehr als 1973 und machte $40 \%$ der gesamten japanischen Einfuhren aus. Als zusätzliches Problem zeichnet sich - sollte aus irgendwelchen Gründen die Strasse von Malakka für Grosstanker gesperrt werden - die Verlängerung der Zufahrtswege vom Persischen Golf ab. Der Kohlebergbau ist rückläufig und immer mehr Gruben werden stillgelegt. Die Erzeugung von Hydroelektrizität stagniert wegen Erreichung der Ausbaugrenze. Der steigende Bedarf von Elektrizität kann nur durch Thermowerke (hauptsächlich mit Oelfeuerung) gedeckt werden:1960 wurden 50\%, 1973 85\% der elektrischen Energie thermisch erzeugt. Absolut hatte sich in dieser Zeit die Elektrizitätsproduktion mehr als vervierfacht, jene von thermischer Elektrizität mehr als versechsfacht. Möglichkeiten der gesteigerten Eigendeckung bestehen allenfalls für Erdöl und Erdgas aus off-shore-Gebieten.
Bei der Betrachtung der Energiestruktur eines Landes darf man nicht bei der Primärenergie stehen bleiben; sie bildet gewissermassen nur das erste Kapitel, weitere betreffen deren Umwandlung in die von den Marktsektoren gewünschte Form (Endenergie) und anschliessend die Nutzung (Nutzenergie). Die Energiebilanz zu deren Aufstellung die OECD vor kurzem Richtlinien erliess - geht von der Anfangsenergie aus, die sich aus Primärenergie und allenfalls eingeführter Sekundärenergie zusammensetzt. Im Falle von Japan können nur geringe Mengen von Erdölprodukten als Sekundärenergie bezeichnet werden, Anfangs - und Primärenergie sind praktisch identisch.

Dem Industriesektor "Ener gie" kommt die Aufgabe zu, die Anfangsenergie in die vom Verbraucher gewünschte Endenergie umzuwandeln. Elektrizitätswerke, Raffinerien und in Japan auch Stadtgaswerke sind damit be schäftigt. Verluste, Eigenverbrauch an Energie und zusätzlich Exporte (einschl. Bunker) führen dazu, dass 1974 die Endenergie in Japan nur noch71, 9\% der Anfangsenergie ausmachte (USA $=69,0 \%$ und West-Europa 62,4\%). Für den relativ hohen Ausnutzungsfaktor ist entscheidend, dass in Japan Erdöl wichtiger als Kohle ist; an der Anfangsenergie macht Erdöl 2/3 $(W-$ Europa $=1 / 2)$ aus und thermische Energie wird hauptsächlich auf der Basis von Erdöl erzeugt. Japan importiert zwar viel Kohle mit einem Drittel Anteil am internationalen Handel steht es an erster Stelle unter den Einfuhrländern; fast die Hälfte kommt heute aus Australien -, doch wird Kohle hauptsächlich in der Schwerindustrie zum reduzierenden Schmelzen von Eisenerz verwendet.

Die von der OECD unterschiedenen Marktsektoren sind: Industrie, Transporte, andere Verbraucher, Nicht-Energieverbrauch. Sowohl in Japan wie in West-Europa haben "Transporte" $11-12 \%$ Anteil. Der Industriesektor nimmt in Japan 33, 8\% und in West-Europa 24,6\% ab. Die Verwendung im Nicht-Energiesektor ist in Japan fast doppelt so gross wie in WestEuropa. Die grössten Energieverluste entstehen bei diesen verschiedenen Verbrauchern,

Prof. Dr. Hans Boesch, Geographisches Institut Universität, Blümlisalpstr. 10, 8006 Zürich. 
Fig. 1 Die japanischen A-Werke. Die drei Großstadtgebiete

Tokyo-Yokohama, Nagoya, Kyoto-Osaka-Kobe sind durch

Punktraster angegeben.

Fig. 2 Die Betriebsperioden der japanischen A-Werke von März 1974 bis Februar 1976

*) Die Code-Zahlen beziehen sich auf die Tabelle bei Fig. 1

Quelle: Japan Times 21. 2. 1976; für 4a 1-3 (Mihama) sind

in dieser Quelle keine Angaben vorhanden.
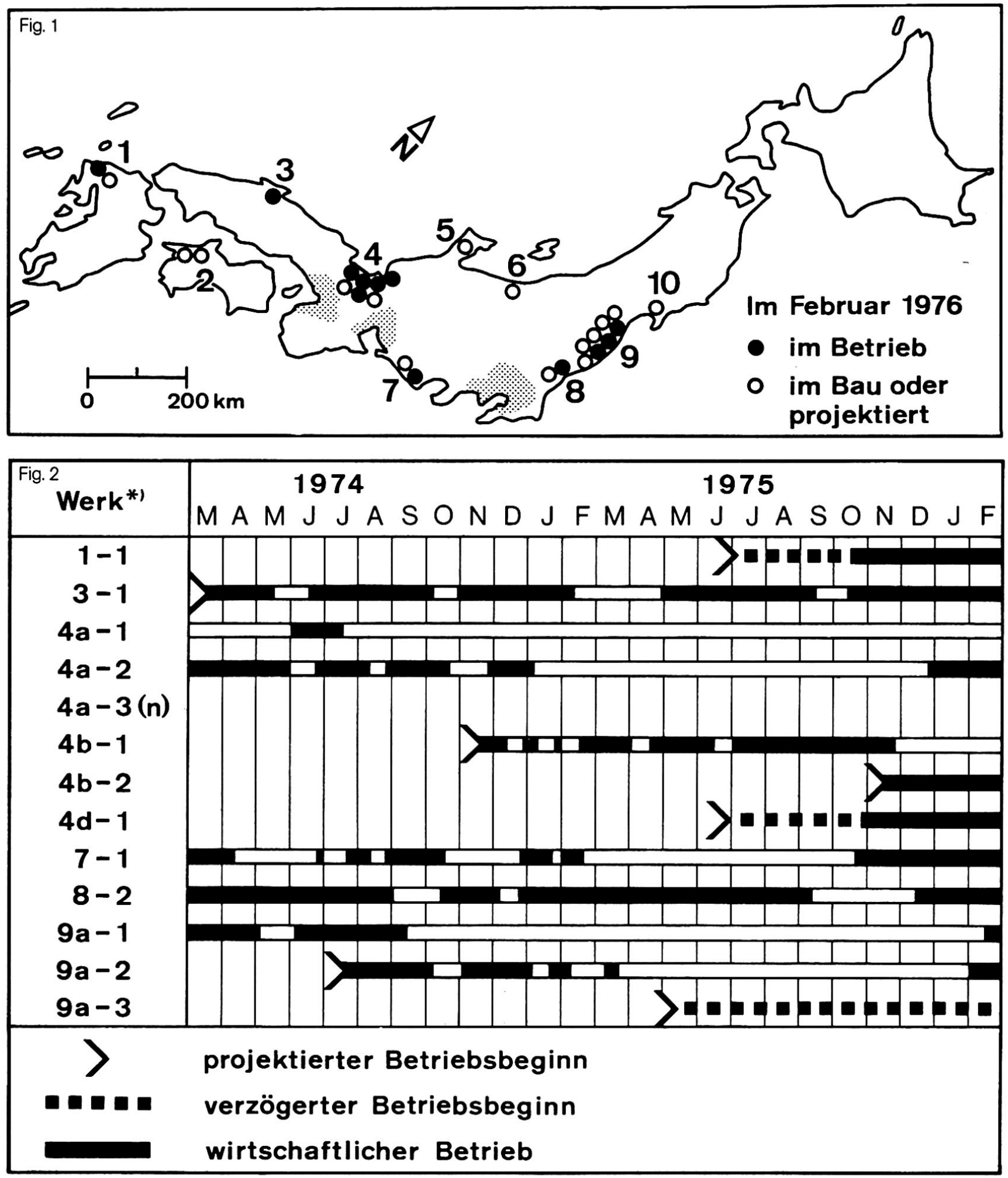


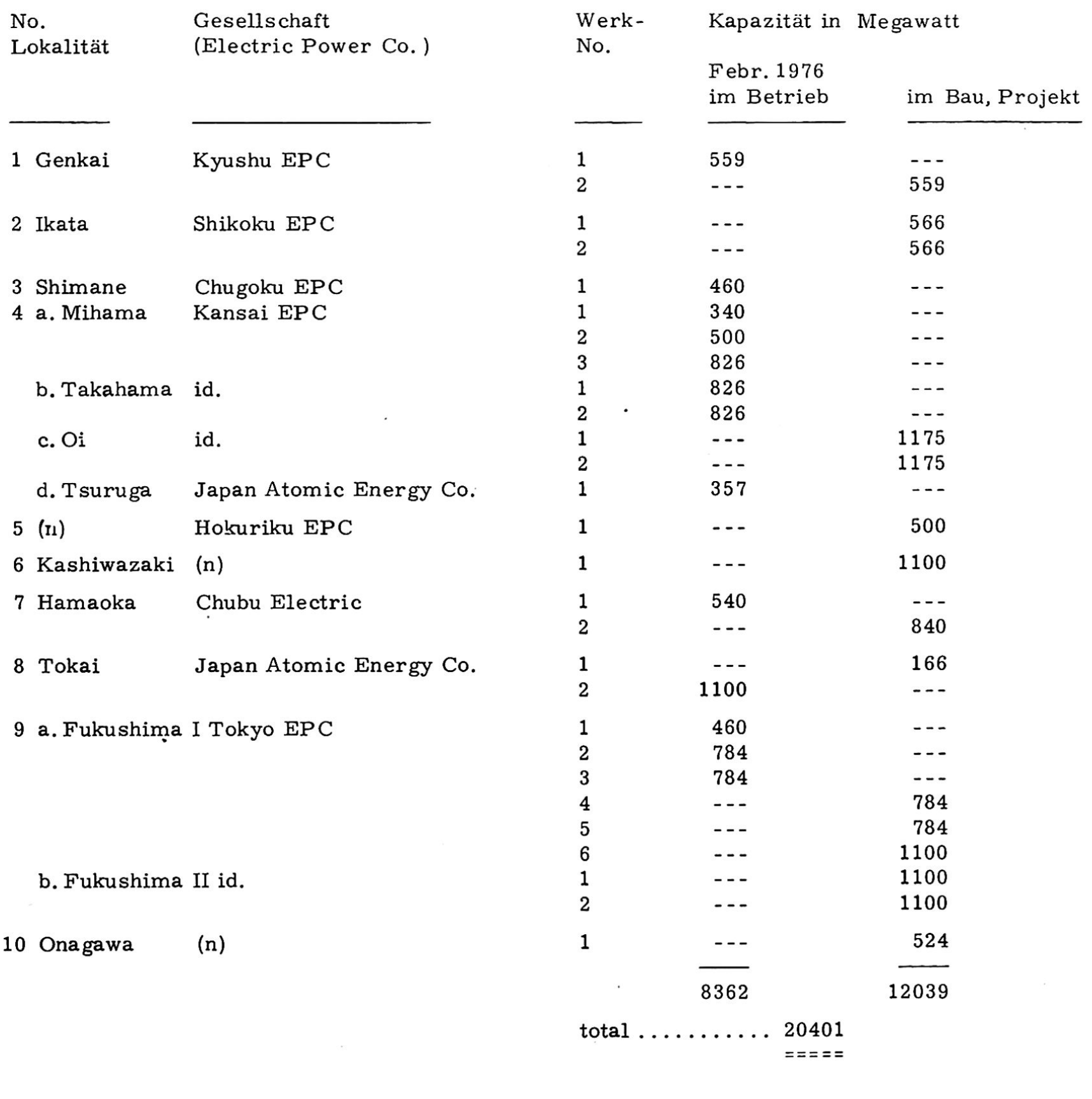

Quelle: Japan Times 21.2. 76 und 22.2.76; $(n)=$ in dieser Quelle keine Angabe vorhanden. 
also zwischen End - und Nutzenergie. Leider geben darüber die für 1974 von der OECD publizierten Energiebilanzen keine Auskunft, weil sie mit der Endenergie abschliessen. Schätzungs weise kann man durch Vergleiche (z. B. Schweiz, USA) mit einem Ausnutzungsfaktor End-:Nutzenergie von etwa $50 \%$ rechnen.

Nachstehend seien einige wenige Besonderheiten der japanischen Verbraucher erwähnt:Ostseitenund Breitenlage bedingen feuchtheisse Sommermonate und darum finden wir in modernen $\mathrm{Ge}$ schäftshäusern und teilweise auch Wohnhäusern üblicherweise Luftkonditionierung; dadurch verschob sich die frühere winterliche Verbrauchsspitze von Elektrizität zum Sommerhalbjahr. Das tägliche Bad am Abend wird seit langem neben Gas und $\mathrm{Holz}$ durch Sonnenenergie ermöglicht. Der traditionelle Holzkohlenraumwärmer "hibachi" ist fast überall, wo keine zentrale Heizung (eine Seltenheit) vorhanden ist, zum mindesten im Aufenthaltsraum durch Petroleumöfen ersetzt worden. Ein japanisches Zimmer erfüllt dank seiner Gestaltung viele Funktionen: Neben Aufenthaltsraum ist es auch Schlafraum und Speiseraum; darum besitzt fast jeder Raum einen Gasanschluss zum Kochen oder für Gasstrahler. Gas wird im allgemeinen mit Gasbomben und nicht über ein Röhrennetz geliefert. In einem späteren Beitrag in dieser Serie werden die veränderten Verkehrsstrukturen untersucht werden, welche in unserem Zusammenhang zu einer Verlagerung auf Elektrizität und Erdölprodukte - und damit zu einem besseren Aus nutzungsfaktor, gleichzeitig aber auch zu einer vergrösserten Abhängigkeit von Einfuhren führten.

Wie andere Industrieländer :suchte Japan nach Alternativen, um die Nachteile der bestehenden Situation zu überwinden. Ebensowenig wie anderswo gibt es hier eine Alternative zum wichtigsten Sektor: Erdöl-Verbrennungsmotor. Lediglich im Sektor Elektrizität glaubt man mittel - und langfristig eine Ausweichmöglichkeit bei der Nuklearenergie (A-Werke) zu sehen.

Natürlich besteht auch bei der Nuklearenergie eine Abhängigkeit bei der Beschaffung der Primärenergie. 1973 schloss Japan mit den USA einen langfristigen Kontrakt über deren Lieferung ab und beteiligte sich gleichzeitig an den Aufbereitungsanlagen für nuklearen Brennstoff.
Abhängig von den USA ist Japan aber auch in hohem Masse in Fragen des technischen "knowhow". Bis 1956 war ihm jegliche Forschung auf dem Gebiete der friedlichen Nutzung von $\mathrm{Nu}-$ klearener gie untersagt. Seither haben Staat und Privatwirtschaft Forschung, technische Entwicklung und wirtschaftliche Anwendung vorwärtsgetrieben. Dabei stiessen sie immer wieder auf den Widerstand der Bevölkerung. "Die zwei Atombombenangriffe im Zweiten Weltkriege haben bei der Bevölkerung eine tiefergreifendere nukleare Allergie hervorgerufen, als man bisher allgemein annahm", heisst es im Area Handbook for Japan, Washington, 1974. Man darf aber auch nicht übersehen, dass die bisherigen Erfahrungen mit der friedlichen Nutzung von Nuklearenergie den Widerstand nicht zu schwächen vermochte. Das erste japanische Schiff mit Nuklearantrieb, die MUTSU, konnte seinerzeit ihren Hafen erst verlassen, als ihr ein Sturm das Durchbrechen einer Blockadekette von Fischerboten ermöglichte; einmal auf offener See musste sie die Fahrt wegen Störungen im Nuklearbereich einstellen und im Frühjahr 1976 suchte man vergeblich eine japanische Hafenstadt, die gewillt gewesen wäre, die MUTSU zur Ueberholung aufzunehmen' Wie Fig. 2 zeigt, war vor allem 1975 ein schlechtes Jahr für die A-Werke; die meisten lagen wegen Schäden grundsätzlicher Natur still. Die verzögerten Betriebsbeginne neuer A-Werke sind ähnlich bedingt. Fig. 1 zeigt die Lage der einzelnen A-Werke, der Figurentext orientiert über weitere Daten. A-Werke sind in der Regel nicht in der Nähe von Bevölkerungs-und Industriezentren zu finden; ob dabei der Sicherheitsfaktor, die Vermeidung von demonstrativem Widerstand und Sabotage oder aber die Absicht, die bestehenden Industrieballungen aufzulockern, leitend war, kann allgemein kaum entschieden werden. Wachsende Opposition und ungelöste technische Probleme führten dazu, dass die Regierung ihre mittelfristigen (bis 1985) Ausbaupläne 1976 von $60000 \mathrm{MW}$ auf $49000 \mathrm{MW}$ kürzte.Seit 1965 schalteten sich die A-Werke als einzige ernstzunehmende Alternative in die japanische Energiewirtschaft ein. 1974 machte ihr Beitrag an die Elektrizitätserzeugung mit $r d$. $20 \mathrm{Mrd}$ kwh erst $4 \%$ aus. 